University of Wollongong

Research Online

Faculty of Engineering - Papers (Archive)

Faculty of Engineering and Information

Sciences

January 2006

\title{
Guided-wave THz time-domain spectroscopy of highly doped silicon using parallel-plate waveguides
}

Rajind Mendis

University of Wollongong, rajind@uow.edu.au

Follow this and additional works at: https://ro.uow.edu.au/engpapers

Part of the Engineering Commons

https://ro.uow.edu.au/engpapers/310

\section{Recommended Citation}

Mendis, Rajind: Guided-wave THz time-domain spectroscopy of highly doped silicon using parallel-plate waveguides 2006.

https://ro.uow.edu.au/engpapers/310

Research Online is the open access institutional repository for the University of Wollongong. For further information contact the UOW Library: research-pubs@uow.edu.au 


\section{Guided-wave THz time-domain spectroscopy of highly doped silicon using parallel-plate waveguides}

\section{R. Mendis}

A novel spectroscopy technique that uses parallel-plate waveguides for the characterisation of highly conductive materials in the terahertz $(\mathrm{THz})$ frequency regime is presented. This guided-wave technique resolves some of the fundamental problems associated with standard $\mathrm{THz}$ time-domain spectroscopy (THz-TDS) as applied to these optically dense materials. The technique is demonstrated by measuring the conductivity of highly phosphorus doped silicon.

Introduction: Ever since undistorted subpicosecond terahertz $(\mathrm{THz})$ pulse propagation was demonstrated using parallel-plate metal waveguides $[1,2]$ there has been considerable interest in these waveguiding structures for $\mathrm{THz}$ applications. These parallel-plate structures have been used to demonstrate two-dimensional interconnect layers [3], sense nanometre water layers [4], study photonic crystals [5], and for building biosensing systems [6]. This Letter describes another novel use of these structures for the characterisation of highly conductive, optically dense materials resolving some of the fundamental problems associated with standard THz time-domain spectroscopy (THz-TDS) [7, 8].

THz-TDS is generally carried out in two configurations, one where the THz beam is transmitted through the sample [7], the other where the beam is reflected off of the sample [8]. The transmission method is not effective for highly conductive materials, as the sample thickness has to be reduced to impractical limits to obtain a measurable signal. In this case, the reflection method is preferred, provided it is possible to discriminate the sample signal from the reference signal. If this is not possible, this would also breakdown. Furthermore, obtaining precise sample and reference positioning for the reflection method is also quite challenging [8]. The guided-wave spectroscopy technique presented here overcomes these problems, and would be ideal for materials such as highly doped semiconductors, superconductors, and conducting polymers. To demonstrate the technique, highly phosphorus-doped silicon ( $\mathrm{Si}$ ) with a carrier density $>10^{18} \mathrm{~cm}^{-3}$ is used as the candidate material.

Experiment: The sample signal was obtained by substituting one of the metal plates in the parallel-plate waveguide $[1,2]$ by the Si wafer, as shown in Fig. 1. The plate separation was $130 \mu \mathrm{m}$ and the propagation length was $5.75 \mathrm{~mm}$. The reference signal was obtained using the usual all-metal parallel-plate waveguide having the same dimensions. Aluminium ( $\mathrm{Al}$ ) was used for the metal. In obtaining these signals, the waveguides were incorporated into a modified THz-TDS setup, similar to previous planar $\mathrm{THz}$ waveguide experiments $[1,2]$.

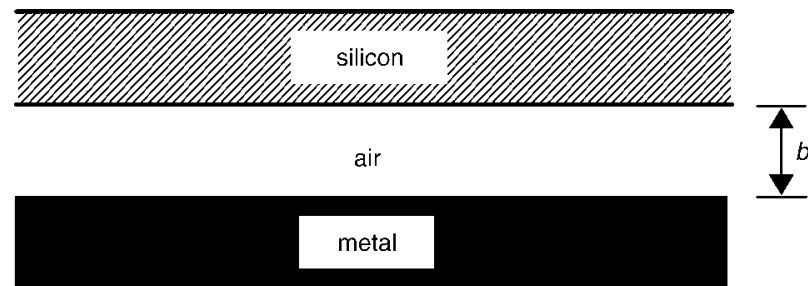

Fig. 1 Cross-sectional view of parallel-plate waveguide

The propagated pulses are shown in Fig. 2, where the reference and sample signals correspond to Figs. $2 a$ and $b$, respectively. The amplitude spectra of these time scans are given in Fig. $3 a$, derived by taking the Fourier transforms. The clean propagated pulses having smooth spectra with no cutoff states, confirm the single TEM-mode nature of propagation $[1,2]$. This is the dominant mode in the parallel-plate waveguide when the input electric field is polarised normal to the plane of the plates [9], as employed here. Realisation of single TEM-mode propagation allows the loss analysis to be carried out using well-known TEM-mode concepts as in the following Section.
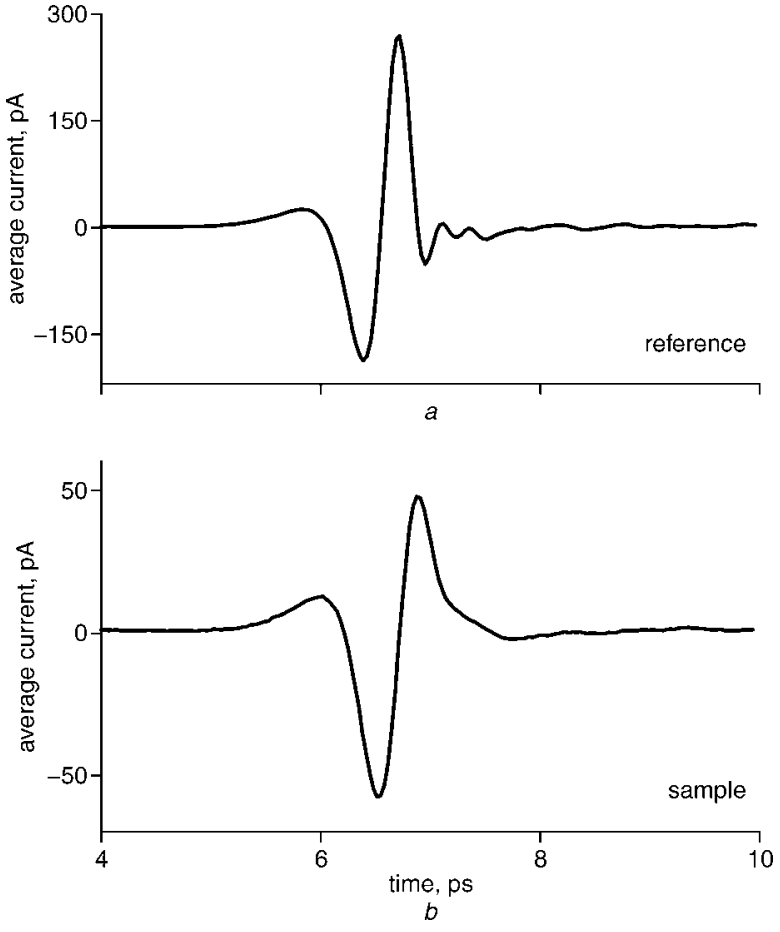

Fig. 2 Propagated pulses

$a$ Reference signal

$b$ Sample signal
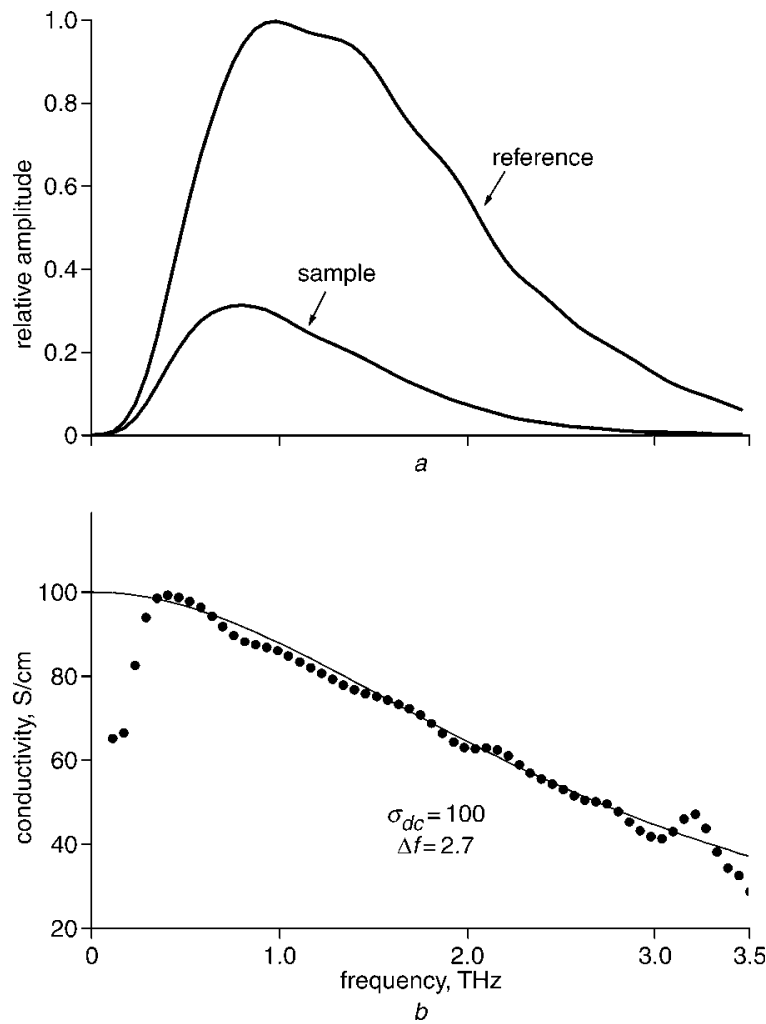

Fig. 3 Amplitude spectra and conductivity of $\mathrm{Si}$

a Amplitude spectra of time scans

$b$ Measured conductivity (dots) and theoretical fit (solid line)

Analysis: The input-output relationship of the singlemode waveguide system can be written in the frequency domain as [1]

$$
E_{\text {out }}(\omega)=E_{\text {in }}(\omega) T C^{2} e^{-j\left(\beta_{z}-\beta_{o}\right) L} e^{-\alpha L}
$$

where $E_{\text {out }}(\omega)$ and $E_{\text {in }}(\omega)$ are the spectral components at frequency $\omega$ of the output (propagated) and input electric fields, $T$ is the transmission coefficient, $C$ is the coupling coefficient, $L$ is the propagation distance, $\alpha$ is the attenuation constant, $\beta_{z}$ is the phase constant, and $\beta_{o}=2 \pi / \lambda_{o}$, where $\lambda_{o}$ is the free-space wavelength. 
The propagation loss introduced by the electrical conductivity of the material forming the two plates, can be expressed as $\alpha=R /\left(\eta_{o} b\right)$, for the usual case where both plates are made of the same material [9]. Here, $R=10.88 \times 10^{-3}\left[10^{7} /\left(\sigma \lambda_{o}\right)\right]^{0.5}$ is the surface resistance, $\eta_{o}$ is the free-space wave impedance, $b$ is the plate separation, and $\sigma$ is the conductivity of the plate material. When the two plates are not made of the same material, the above expression for $\alpha$ can be modified to read as

$$
\alpha=\left(R_{\mathrm{Al}}+R_{\mathrm{Si}}\right) /\left(2 \eta_{o} b\right)
$$

since the overall loss is the sum of the losses due to each plate. Here, $R_{\mathrm{Al}}$ and $R_{\mathrm{Si}}$ stand for the surface resistances for $\mathrm{Al}$ and $\mathrm{Si}$.

The difference between $\alpha$ 's for the sample and reference signals can be obtained by applying (1) to their spectral components, taking the complex ratio, and then extracting the amplitude information. Looking at the same difference using (2), the conductivity of $\mathrm{Si}$ is solved to be

$$
\sigma_{\mathrm{Si}}=\sigma_{\mathrm{Al}}\left[1-\sqrt{\sigma_{\mathrm{Al}} \lambda_{o} / 10}\left(\eta_{o} b / 5.44 L\right) \ln \left(E_{\text {out } 2} / E_{\text {out } 1}\right)\right]^{-2}
$$

where $\sigma_{\mathrm{Al}}$ is the conductivity of $\mathrm{Al}$, and $E_{\mathrm{out} 2}$ and $E_{\text {out1 }}$ correspond to the sample and reference signals, respectively. By knowing the conductivity of $\mathrm{Al}$, the conductivity of $\mathrm{Si}$ can be derived via the sample and reference signals using (3). This is plotted (dots) in Fig. $3 b$, where $\sigma_{\mathrm{Al}}$ was taken to be constant and equal to the DC value of $3.96 \times 10^{7} \mathrm{~S} / \mathrm{m}$, since $\mathrm{Al}$ has a flat frequency response in this range. The validity of this measurement is conditional upon the thickness of the $\mathrm{Si}$ wafer being greater than the effective skin depth in Si. The skin depth can be estimated using the well-known expression, $\delta \simeq[2 /(\omega \mu \sigma)]^{0.5}$, where $\mu$ is the permeability. For $\sigma=100 \mathrm{~S} / \mathrm{cm}$ (upper-limit of measurement), $\delta \simeq 7 \mu \mathrm{m}$ at $0.5 \mathrm{THz}$, and decreases with increasing frequency. As the wafer thickness was $127 \mu \mathrm{m}$, this condition was well satisfied.

The experimentally deduced conductivity can be compared to the simple theoretical Drude model [7], where the frequency dependence of the real part of the complex conductivity (as measured here) is given by $\sigma=\sigma_{\mathrm{dc}} \Gamma^{2} /\left(\omega^{2}+\Gamma^{2}\right)$, with the damping rate $\Gamma=2 \pi \Delta f$, where $\Delta f$ is the collision frequency. The DC conductivity $\sigma_{\mathrm{dc}}=e \mu N$, with the mobility $\mu=e /\left(m^{*} \Gamma\right)$, where $e$ is the electron charge, $N$ is the carrier density, and $m^{*}$ is the effective mass. Fig. $3 b$ shows this comparison, where the solid line represents the theoretical curve, with the fitting parameters, $\sigma_{\mathrm{dc}}=100 \mathrm{~S} / \mathrm{cm}$ and $\Delta f=2.7 \mathrm{THz}$. These parameters predict $N=1.6 \times 10^{18} \mathrm{~cm}^{-3}$ and $\mu=398 \mathrm{~cm}^{2} \mathrm{~V}^{-1} \mathrm{~s}^{-1}$, which are found to be consistent with empirical mobility-density curves [10]. The measured conductivity is much higher than has been previously recorded for doped $\mathrm{Si}$ in the $\mathrm{THz}$ regime [7], representative of the high doping concentration. The minor fluctuations in the experimental curve can be attributed to surface-specific conditions such as roughness, oxidation, and water adsorption. The substantial deviations at the high and low frequency ends are due to low signal content.
Conclusion: A guided-wave $\mathrm{THz}$ time-domain spectroscopy technique that uses parallel-plate waveguides for the study of highly conductive materials is presented. Although demonstrated here using highly doped $\mathrm{Si}$, this technique can be applied to virtually any material that is optically dense in the $\mathrm{THz}$ regime owing to high conductivity. It serves as a powerful tool for characterising such 'opaque' materials where standard THz-TDS would fail.

Acknowledgment: The author is grateful to D. Grischkowsky for guidance and support.

(C) IEE 2006

Electronics Letters online no: 20063418

27 September 2005 doi: 10.1049/el:20063418

R. Mendis (Faculty of Engineering, University of Wollongong, Wollongong, NSW 2522, Australia)

E-mail: rajind@vow.edu.au

\section{References}

1 Mendis, R., and Grischkowsky, D.: 'Undistorted guided-wave propagation of subpicosecond terahertz pulses', Opt. Lett., 2001, 26, pp. $846-848$

2 Mendis, R., and Grischkowsky, D.: 'THz interconnect with low-loss and low-group velocity dispersion', IEEE Microw. Wirel. Compon. Lett., 2001, 11, pp. 444-446

3 Coleman, S., and Grischkowsky, D.: 'A THz transverse electromagnetic mode two-dimensional interconnect layer incorporating quasi-optics', Appl. Phys. Lett., 2003, 83, pp. 3656-3658

4 Zhang, J., and Grischkowsky, D.: 'Waveguide terahertz time-domain spectroscopy of nanometer water layers', Opt. Lett., 2004, 29, pp. 1617-1619

5 Jian, Z., Pearce, J., and Mittleman, D.M.: 'Defect modes in photonic crystal slabs studied using terahertz time-domain spectroscopy', Opt. Lett., 2004, 29, pp. 2067-2069

6 Nagel, M., Bolivar, P.H., and Kurz, H.: 'Modular parallel-plate THz components for cost-efficient biosensing systems', Semicond. Sci. Technol., 2005, 20, pp. S281-S285

7 Jeon, T.I., and Grischkowsky, D.: 'Nature of conduction in doped silicon', Phys. Rev. Lett., 1997, 78, pp. 1106-1109

8 Jeon, T.I., and Grischkowsky, D.: 'Characterisation of optically-dense, doped semiconductors by reflection THz time domain spectroscopy', Appl. Phys. Lett., 1998, 72, pp. 3032-3034

9 Marcuvitz, N.: 'Waveguide handbook' (Peter Peregrinus, London, 1993)

10 Baccarani, G., and Ostaja, P.: 'Electron mobility empirically related to the phosphorus concentration in silicon', Solid-State Electron., 1975, 18, pp. $579-580$ 